$18^{\text {th }}$ World Conference on Non destructive Testing, 16-20 April 2012, Durban, South Africa

\title{
Ultrasonic Multi-Skip Tomography for Pipe Inspection
}

\author{
Arno VOLKER ${ }^{1}$, Rik $_{\text {VOS }}^{1}$ Alan HUNTER $^{1}$ \\ ${ }^{1}$ TNO, Stieltjesweg 1, Delft, The Netherlands, \\ Phone: +31 (0)88 86 66292, e-mail: arno.volker@tno.nl
}

\begin{abstract}
.
The inspection of wall loss corrosion is difficult at pipe support locations due to limited accessibility. However, the recently developed ultrasonic Multi-Skip screening technique is suitable for this problem. The method employs ultrasonic transducers in a pitch-catch geometry positioned on opposite sides of the pipe support. Shear waves are transmitted in the axial direction within the pipe wall, reflecting multiple times between the inner and outer surfaces before reaching the receivers. Along this path, the signals accumulate information on the integral wall thickness (e.g., via variations in travel time). The method is very sensitive in detecting the presence of wall loss, but it is difficult to quantify both the extent and depth of the loss. If the extent is unknown, then only a conservative estimate of the depth can be made due to the cumulative nature of the travel time variations. MultiSkip tomography is an extension of Multi-Skip screening and has shown promise as a complimentary follow-up inspection technique. In recent work, we have developed the technique and demonstrated its use for reconstructing high-resolution estimates of pipe wall thickness profiles. The method operates via a model-based full wave field inversion; this consists of a forward model for predicting the measured wave field and an iterative process that compares the predicted and measured wave fields and minimizes the differences with respect to the model parameters (i.e., the wall thickness profile). This paper presents our recent developments in Multi-Skip tomographic inversion, focusing on the initial localization of corrosion regions for efficient parameterization of the surface profile model and utilization of the signal phase information for improving resolution.
\end{abstract}

Keywords: Multi-Skip, wave field inversion, tomography, wall thickness profile, corrosion, support

\section{Introduction}

Pipe supports are locations where corrosion quite frequently occurs due to the ingress of water. Inspection of these locations is a difficult task because of accessibility issues. Recently a new screening method has been developed, called Multi-Skip (or M-Skip ${ }^{\circledR}$ ) ultrasonics[1]. This method uses shear waves at typical incidence angles between 45 and 70 degrees that reflect multiple times in the pipe wall. This allows the transmission of shear waves over long distances that gather information about the wall thickness during propagation. Wall thickness loss manifests itself as a change of travel time. A circumferential scan is used to detect any wall thickness reduction around the pipe at the support location. This method turns out to be very sensitive to detecting the presence of wall thickness loss. However, the travel time changes are proportional to the integral wall thickness loss, while for integrity assessment the deepest corrosion pit is of interest. In this paper we introduce an extension of the Multi-Skip method, called Multi-Skip tomography. This method allows us to construct a wall thickness profile under a pipe support, at a certain circumferential position.

\section{Ray approximation versus full-wavefield approach}

A high-frequency ray propagation model can be employed to simplify the tomography problem. Furthermore, the interaction of the rays with the surface profile can be simplified by assuming that the deviations from a planar surface are small such that the defect can be modeled as a "phase screen". In this approximation, the surface profile deviations are modeled as a reflection coefficient profile that applies time delay perturbations only (or linear phase shifts) to the propagating signals, ignoring the geometric perturbations of the rays. For surface profile 
deviations $\Delta z(x)$ with respect to lateral position $x$, the time delay perturbations for the phase screen are given by

$$
\Delta t(x)=(2 \Delta z(x) \cos \theta) / c
$$

where $c$ is the propagation speed, and $\theta$ is the angle of incidence of the ray. By considering the time delays $\Delta t_{m n}^{(k)}$ for all source and receiver pairs (denoted by indices $m$ and $n$ ) and for all skips (denoted by the superscript $k$, which runs from 1 to $K$ ), one can establish a system of linear equations:

$$
\mathbf{A} \Delta \mathbf{z}=\Delta \mathbf{t},
$$

where $\Delta \mathbf{t}=\left(\Delta t_{11}^{(1)}, \cdots, \Delta t_{M N}^{(1)}\left|\Delta t_{11}^{(2)}, \cdots, \Delta t_{M N}^{(2)}\right| \cdots \mid \Delta t_{11}^{(K)} \cdots, \Delta t_{M N}^{(K)}\right)^{\mathrm{T}}$ is a vector of the time delay perturbations, $\Delta \mathbf{z}=\left(\Delta z_{1}, \Delta z_{2}, \cdots, \Delta z_{P}\right)^{\mathrm{T}}$ is a parameterization of the surface profile deviations into $P$ discrete values, and $\mathbf{A}$ is a $(M N K \times P)$ matrix of coefficients.

The time delay perturbations can be measured from the collected data by cross-correlating the signals acquired for each source / receiver pair $m n$ and isolated skip $k$ with the signals expected for a planar surface profile, $\Delta z_{0}(x)=0$. The system of equations can then be inverted to obtain the surface profile deviations via a least-squares solution, i.e.,

$$
\Delta \tilde{\mathbf{z}}=\underset{\Delta z}{\arg \min }(\mid \mathbf{A} \Delta z-\Delta \hat{\mathbf{t}}))
$$

where $\boldsymbol{\Delta} \hat{\mathbf{t}}$ are the time delays measured from the data. We employ a weighted least-squares solution and use the correlation coefficient as a weighting factor.

A modeled example solution is shown in Figure 1 for a flat-bottomed defect of depth $1 \mathrm{~mm}$, using a single source, a wideband pulse from $400 \mathrm{kHz}-1200 \mathrm{kHz}$, and 256 receivers. The surface profile was estimated using the approach described above. Here, measured time delays with correlation coefficients less than 0.95 were excluded from the solution. The estimated surface profile is shown to agree with the actual profile in terms of the location, width, and depth of the defect. However, it also exhibits significant noise. Moreover, the solutions are worse for deeper defects and at lower frequencies due to the violation of the phase screen and high-frequency assumptions. This limits the applicability of the technique and has motivated the use of a more complicated full-wave field solution detailed in the following sections. It is possible, however, that this simple technique could be used as a first stage to localize the defect and to refine the surface parameterization for the full-wave field inversion. 


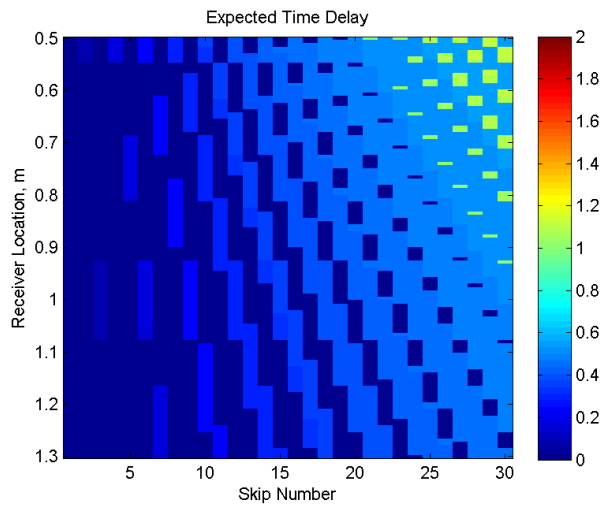

a)
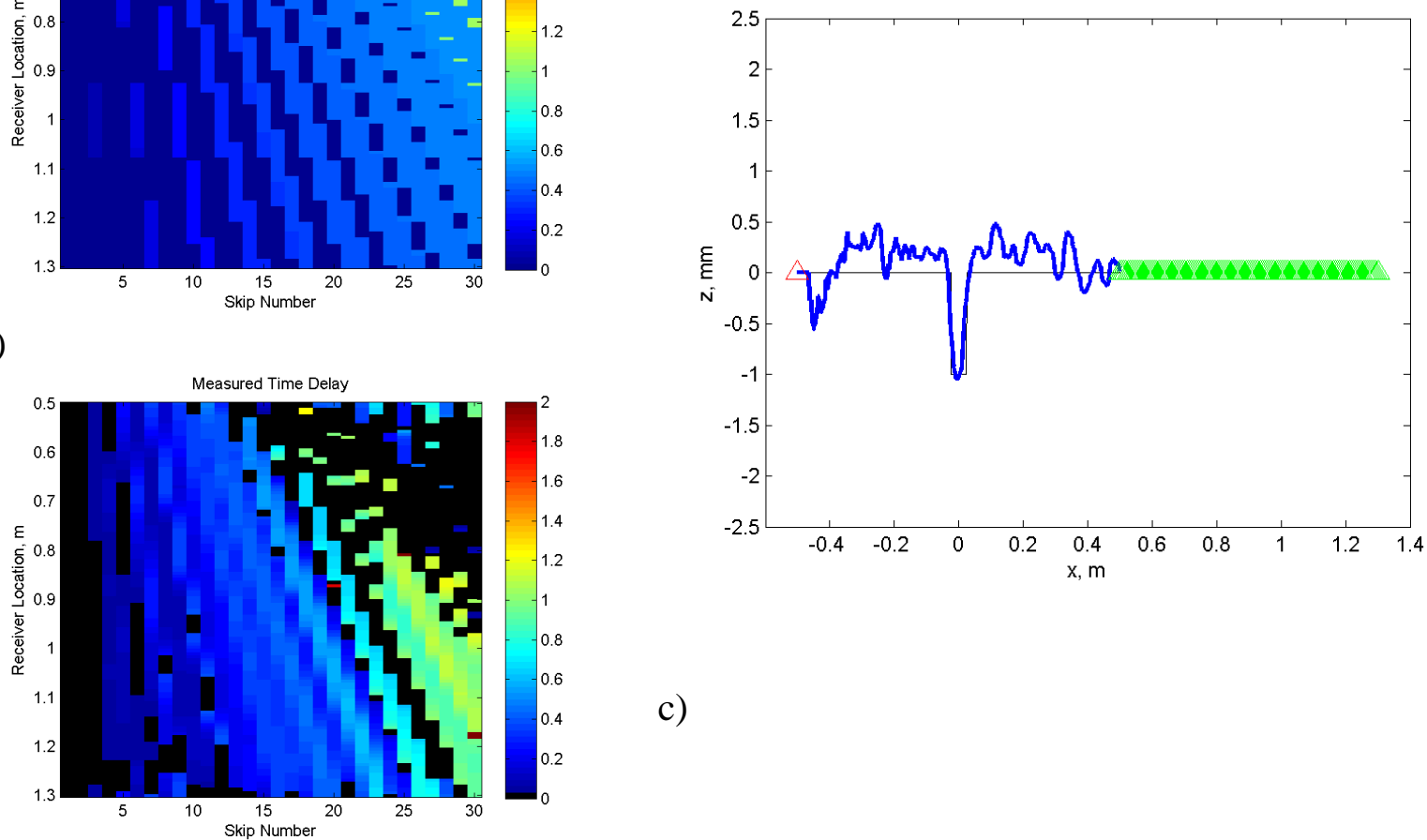

c)

b)

Figure 1 - Demonstration of topographic inversion of the surface profile using an assumed ray propagation model and a phase screen defect model. The expected arrival time differences (defect - no defect) are shown in (a) and the measured differences are shown in (b); black regions indicate a correlation coefficient below the threshold of 0.95 . The actual and estimated surface profiles are shown in (c) in black and blue, respectively, where the source position is indicated by a red marker and the receiver positions are indicated by the green markers.

\section{Forward wave field model}

The forward modeling procedure is conceptually explained in Figure 2, using a wave field operator formulation [2]. In Figure 2, $\mathbf{S}\left(z_{0}\right)$ is the emitted wave field from a wedge transducer at the steel-wedge interface. This operator includes elastic transmission effects from the wedge into the steel. The wave field propagation operator $\mathbf{W}^{+}\left(z_{m}, z_{0}\right)$, extrapolates the wave field from the top surface to the back wall. Note that zm may vary with the lateral coordinate. The wave field is reflected from the back wall, where $\mathbf{R}^{+}\left(z_{m}\right)$ describes the angle dependent reflection. The upward propagation operator $\mathbf{W}^{-}\left(z_{0}, z_{m}\right)$ propagates the waves to the outer surface at z0 and again, z0 may vary laterally. Part of the wave field is detected by a wedge transducer, described by $\mathbf{D}\left(z_{0}\right)$ and another part is reflected back, described by $\mathbf{R}^{-}\left(z_{0}\right)$. Each pass through the feedback loop yields another, higher order skip. The wave fields and operators are formulated in the temporal frequency domain. 
The inversion model is parameterized with $z_{0}=z_{0}(x)$ and $z_{m}=z_{m}(x)$, where $\mathrm{x}$ is the lateral coordinate. The data matrix for primary reflections, i.e., ignoring multiple reflections can be written as:

$$
\mathbf{P}\left(z_{0}\right)=\mathbf{D}\left(z_{0}\right) \mathbf{W}^{-}\left(z_{0}, z_{m}\right) \mathbf{R}^{+}\left(z_{m}\right) \mathbf{W}^{+}\left(z_{m}, z_{0}\right) \mathbf{S}\left(z_{0}\right) .
$$

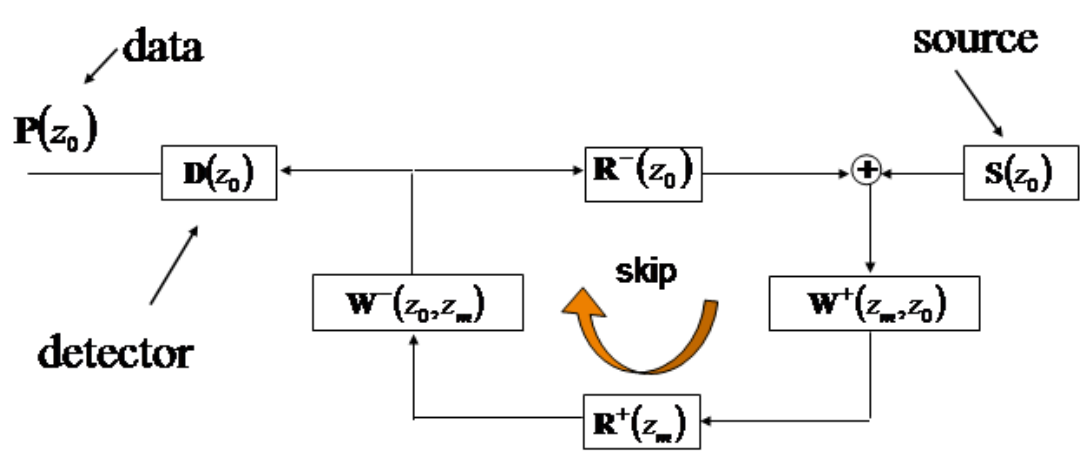

Figure 2 Schematic illustration of wave propagation model for elastic, anisotropic media. The wave field is generated by an arbitrary source array $(\mathrm{S})$ that describes both the directivity and the signal spectrum. The wave field propagates down $\left(\mathrm{W}^{+}\right)$and gets reflected $\left(\mathrm{R}^{+}\right)$, where the reflection operator describes general angle dependent reflectivity. The second propagation $\left(\mathrm{W}^{-}\right)$is back to the surface and the detector response is expressed by D. Multiple reflections are generated by the feedback loop.

\section{Wave field inversion scheme}

Wave field inversion of Multi-Skip measurements relies on an iterative inversion scheme. An objective function is defined, which is minimized iteratively. In our approach, the objective function consists of three separate terms, the difference between the measured $P_{m}\left(z_{0}\right)$ and forward modeled wave field $P_{c}\left(z_{0}\right)$, the surface parameters $z\left(x_{i}\right)$ and a smoothness constraint (second derivative). These terms are assigned relative scaling factors:

$$
F=\left[\left|P_{m}\left(z_{0}\right)\right|-\left|P_{c}\left(z_{0}\right)\right| \quad \eta_{1} z\left(x_{i}\right) \quad \eta_{2} \frac{\partial^{2} z}{\partial x^{2}}\right]
$$

In order to update the parameters, partial derivatives are calculated:

$$
\mathbf{J}=\frac{\partial F}{\partial x_{i}}
$$

where $\mathbf{J}$ is the jacobian matrix. The last two terms in the objective function play an important role in regularizing the inversion problem. To illustrate the influence of these two regularization terms on the performance of the inversion a $20 \mathrm{~mm}$ wide flat bottom hole with a depth of $6 \mathrm{~mm}$ is modeled. First, we evaluate the influence of the smoothness constraint (second regularization term); the sparseness term (first regularization term) is ignored. The scale parameter $\eta_{2}$ is set in such a way that the contributions are $0 \%, 1 \%$ and $10 \%$ of the difference 
in wave field amplitude. The result is shown in Figure 3. All results shown here are for the 8th skip and a frequency of $400 \mathrm{kHz}$. Twelve source positions are modeled on the left hand size of the defect. The receiving transducer is scanned from $\mathrm{x}=0.5 \mathrm{~m}$ to $\mathrm{x}=1.25 \mathrm{~m}$, with a step size of $0.5 \mathrm{~mm}$. The wall thickness is $33 \mathrm{~mm}$.

Using no smoothness regularization (0\%), the inversion result contains a lot of artifacts. Adding $1 \%$ of regularization, significantly improves the result, both the shape and the depth are much more accurate. If more regularization is added (10\%), the influence becomes too strong and this yields a solution that is too smooth. Additionally, more oscillations left and right of the actual defect are observed. In terms of depth sizing, the performance is similar.

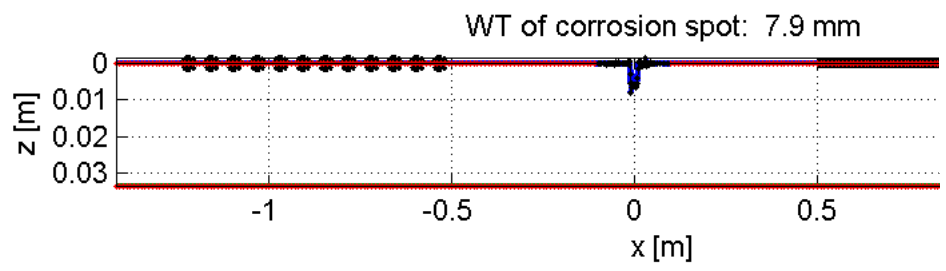

a)

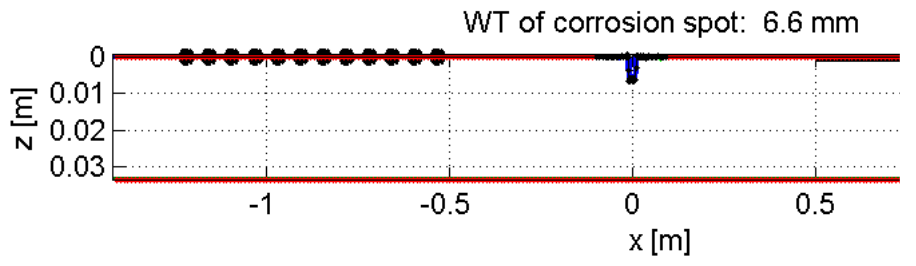

b)
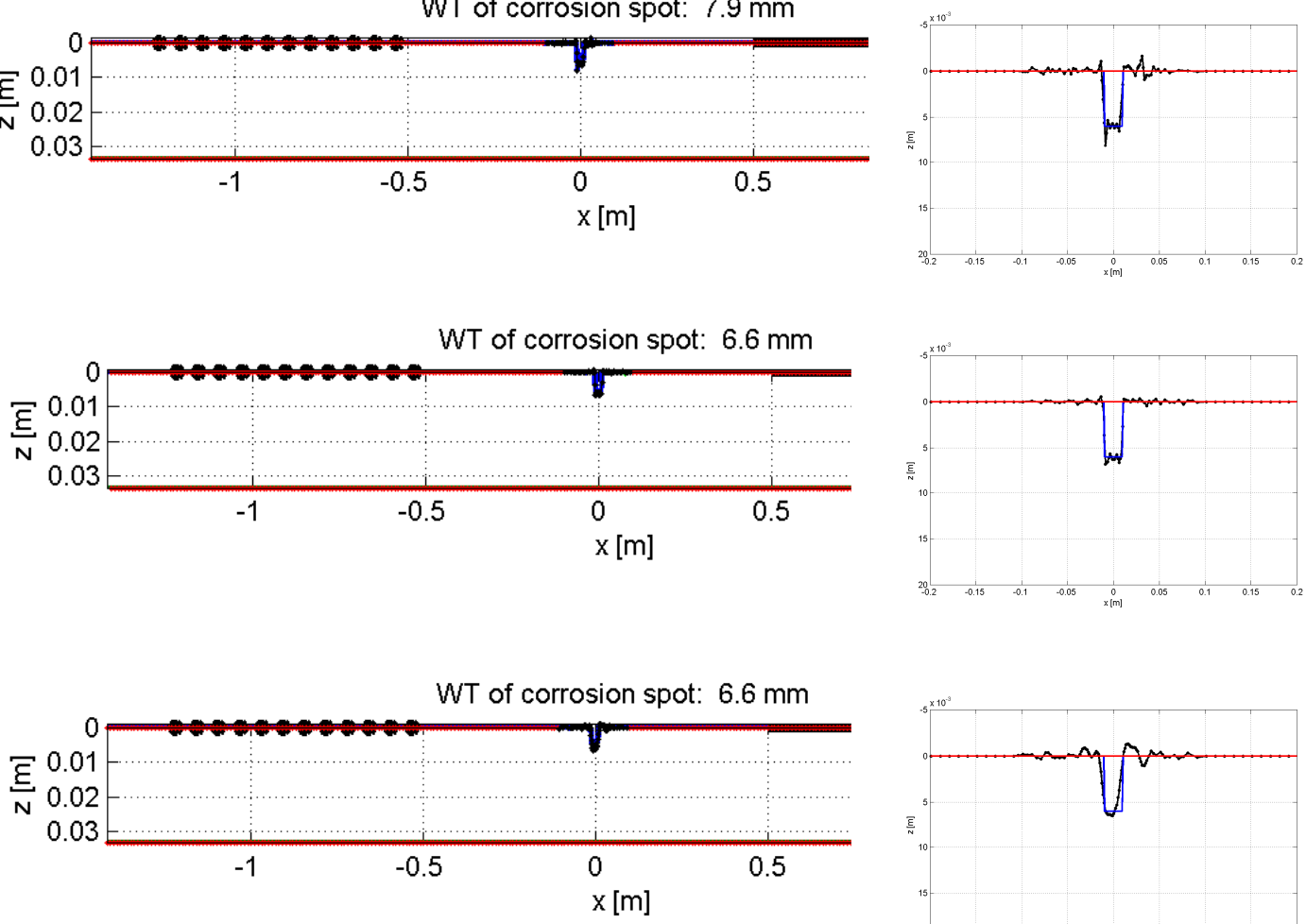

c)

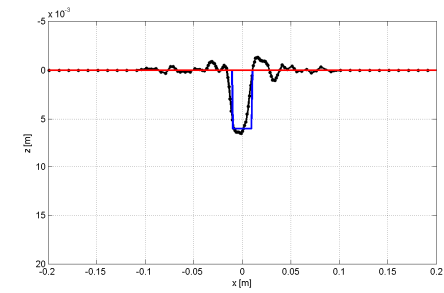

Figure 3 Influence of second regularization term, the relative magnitude equals, a) $0 \%$, b) $1 \%$ and c) $10 \%$. The geometry consists of 12 sources locations and with the receiver transducer a scan is performed from $\mathrm{x}=0.5 \mathrm{~m}$ to $\mathrm{x}=1.25 \mathrm{~m}$, with a step size of $0.5 \mathrm{~mm}$

Similarly we evaluate the influence of the first (sparseness) regularization term. This term suppresses parameters that are poorly resolved in the misfit between the measured and model data. Effectively this yields a sparse solution, suppressing insignificant parameter values. Figure 4 shows the inversion results with a 10\% smoothness constraint (a) and with a $10 \%$ sparseness constraint (b).
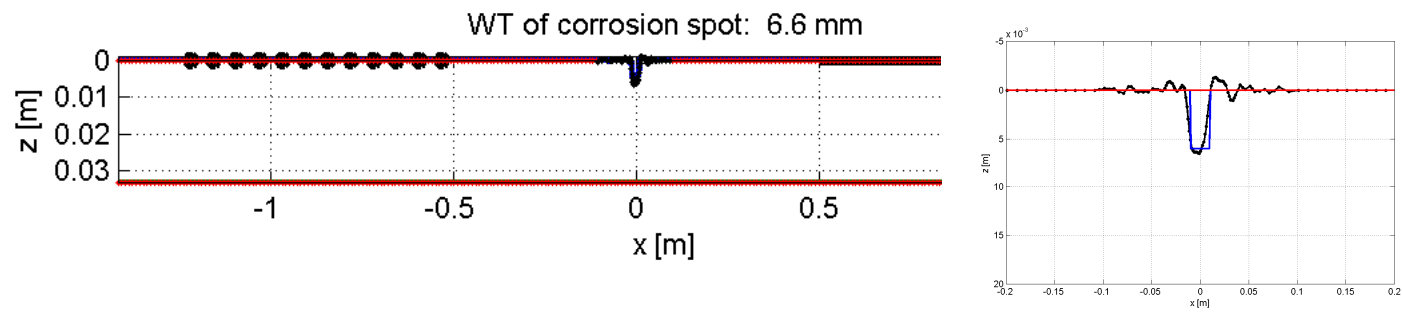

a) 

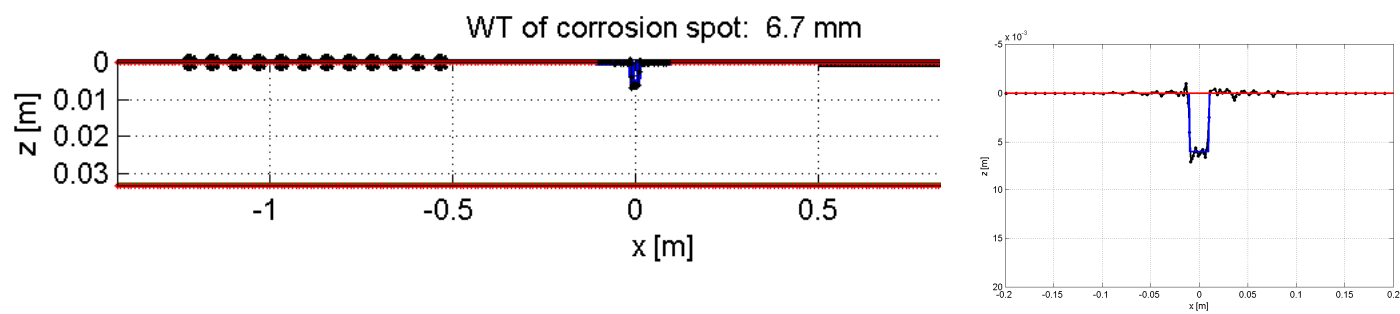

b)

Figure 4 Comparison of the influence of the smoothness term and the sparse term to regularize the inversion problem. a) $10 \%$ smoothness added to the objective function, b) $10 \%$ sparseness added to the objective function

As expected the sparseness constraint suppresses irrelevant parameters. In terms of quality, this result is quite similar to a $1 \%$ smoothness constraint. To update the parameter, the Jacobian matrix is calculated by varying each parameter individually. Based on the Jacobian matrix, the resolution matrix can be calculated as follows:

$$
\mathbf{R}=\mathbf{J}^{T} \mathbf{J},
$$

where ' $\mathrm{T}$ ' indicates the transposed matrix. Essentially the resolution matrix shows how well each parameter is resolved and whether there are correlations with other parameters.

Correlations with other parameters are undesired since it increases instability in the iterative optimization. Ideally the resolution matrix equals a diagonal matrix, where all elements on the main diagonal have the same value.

The resolution matrices for 0\%, 1\% and 10\% sparseness constraint are shown in Figure 5. As can be seen in Figure 5a there are strong side bands, which are likely to cause artifacts. Adding some regularization clearly improves the problem, as shown in Figure 5b. Figure 5c shows the resolution matrix for a $10 \%$ sparseness constraint, which is nearly ideal.

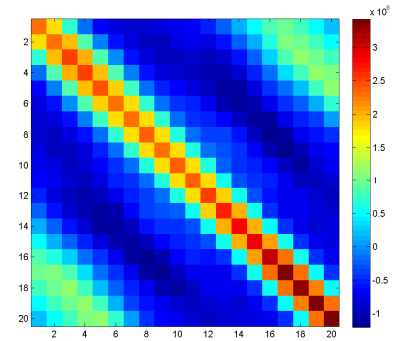

a)

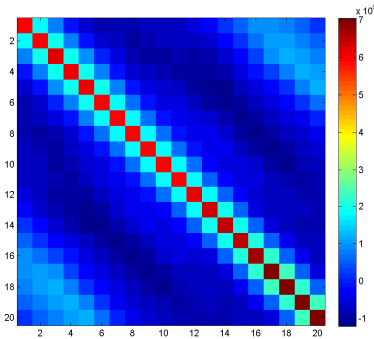

b)

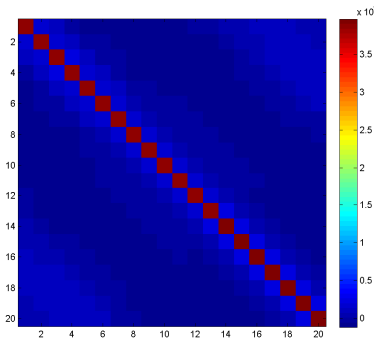

c)

Figure 5 Resolution matrices showing the effect of regularization with a sparseness constraint: a) no constraint b) $1 \%$ sparseness constraint, c) $10 \%$ sparseness constraint.

The resolution matrices for a smoothness constraint are shown in Figure 6, again a 0,1 and 10\% smoothness constraint was added. The smoothness constraint is realized by calculating the second derivative of the parameters, i.e., the shape of the outer pipe surface. This inherently introduces correlations with neighboring parameters as can be seen from the resolution matrices shown in Figure 6b and c. 


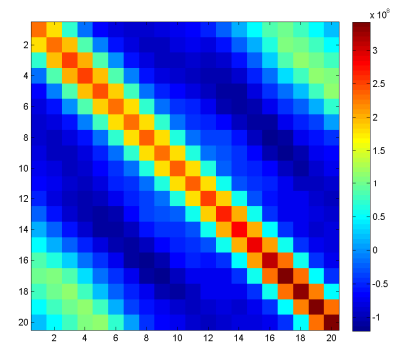

a)

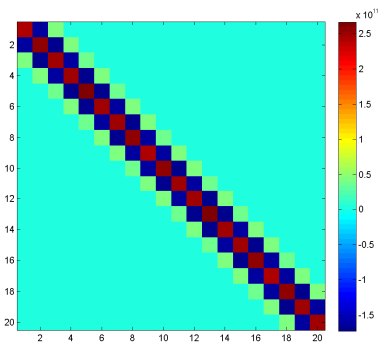

b)

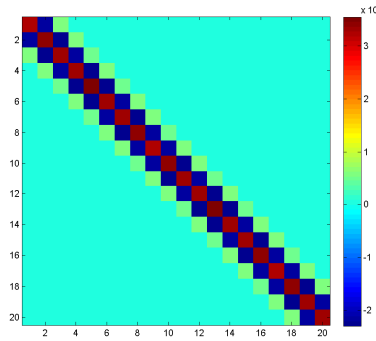

c)

Figure 6 Resolution matrices showing the effect of regularization with a smoothness constraint, a) no smoothness constraint, b) $1 \%$ smoothness constraint, c) $10 \%$ smoothness constraint.

Already $1 \%$ smoothness constraint added to the objective function has a significant effect on the resolution matrix. The smoothness constraint is a powerful method to regularize the inversion problem. We have seen however that too much influence of the smoothness constraint yields oscillations in the solution.

In our inversion scheme, we start at a low frequency and low skip order. Gradually increasing the skip order and the frequency allows for a high resolution reconstruction. This requires an adaptive parameterization. The resolution matrix will be used further to assess whether local refinement in case of defects will improve the reliability of the solution.

\section{Conclusions and future work}

Multi-Skip tomography is a powerful method to calculate a high resolution wall thickness profile at inaccessible locations. Skipping shear waves ensure a high resolution. Due to the nature of the wave propagation, high frequency approximations do not provide sufficient accuracy to accurately reconstruct the wall thickness profile. Our results indicate that with an appropriate wave field modeling scheme, as part of a tomographic reconstruction kernel, an accurate and high resolution wall thickness profile can be reconstructed.

We introduced a conceptual propagation model, which essentially describes all relevant aspects of wave propagation, including elastic angle dependent reflectivity. In our approach we compare a measured wave field to a predicted field using this wave field modeling concept. The objective function consists of the difference between the measured and modeled wave field, supplemented with regularization terms. We have shown the added value of proper regularization. Additionally, the resolution matrix can be used to assess the effect of a specific type of regularization. Moreover, the resolution matrix will be used during further development to assess whether further adaptive refinement of parameters adds additional information.

\section{References}

1. S F Burch, N J Collett, S Terpstra and M V Hoekstra, M-skip: a quantitative technique for the measurement of wall loss in inaccessible components, Insight Vol 49 No 4 April 2007

2. A.J. Berkhout, Seismic migration; A. Theoretical aspects, Elsevier sciences publishers, Amsterdam, 1985.. 